\title{
PROPOSTA DE METODOLOGIA DE AGREGAÇÃO DE ATRIBUTOS E PONDERAÇÃO DE VALORES PARA AVALIAÇÃO DA SIGNIFICÂNCIA DE IMPACTOS AMBIENTAIS
}

\section{PROPOSAL OF METHODOLOGY OF AGGREGATION OF ATTRIBUTES AND WEIGHING VALUES FOR ASSESSMENT OF THE SIGNIFICANCE OF ENVIRONMENTAL IMPACTS}

\section{Isabela Coelho Moreira}

Universidade Federal de Minas Gerais - Campus Pampulha - Escola de Engenharia, Belo Horizonte, MG - Brasil. E-mail: isabelacoelhomoreira@hotmail.com

\begin{abstract}
RESUMO
Este trabalho apresenta uma proposta de metodologia quantitativa para avaliação da significância de impactos ambientais em Estudo de Impactos Ambientais (EIAs) em conformidade à Lei 6.938/1981. Realizaram-se pesquisas em artigos científicos, EIAs, legislações ambientais federais e estaduais. O trabalho desenvolveu um método de agregação de atributos e ponderação de valores numéricos, criou uma fórmula aplicada por meio de um algoritmo e propõe a apresentação dos resultados em uma matriz. Os resultados foram testados e comparados a uma AIA (Análise de Impacto Ambiental) com método qualitativo. Verificou-se que o método quantitativo foi aplicável, trazendo, porém, resultados mais severos, com níveis de significância maiores. Concluiu-se que a junção de métodos quali e quantitativos para a AIA, tornam esta prática mais próxima da realidade, para proposição de ações de controle ambiental.
\end{abstract}

Palavras-chave: Avaliação de Impacto Ambiental. Significância de Impactos. Agregação de Atributos. Ponderação de Valores.

\footnotetext{
ABSTRACT

This paper proposes a quantitative methodology for assessment of the significance of environmental impact on Environmental Impact Statement (EIS) in accordance to Law 6.983/1981. Studies have been made in scientific articles, EISs, Federal and State Environmental Laws. This work developed a method of aggregation of attributes and weighing of numeric values, created a formula applied by an algorithm and proposes to present the results in a matrix. The results were tested and compared to an EIS with a quantitative method. The quantitative method was verified as applicable and furthermore showed more severe results with higher significance. It was concluded that the addition of qualitative and quantitative methods for the EIS, make this practice closer to reality proposition for environmental control measures.

Keywords: Environmental Impact Assessment. Significance of Impacts. Aggregation of Attributes. Weighing of Values.
} 


\section{INTRODUÇÃO}

Diante da evolução das leis ambientais para o desenvolvimento socioeconômico e ambiental do país, constata-se que o Sistema Nacional de Meio Ambiente (SISNAMA), ainda é um sistema com lacunas, principalmente em relação ao ato de fiscalização e controle. Fato apontado por Agnes et al. (2009) ao afirmar que o SISNAMA apresenta sobreposições e problemas de aplicação da legislação ambiental, devido à impasses gerados por interesses conflitantes. Milaré e Coimbra (2004) ratificou esse ponto de vista ao apontar que há um desajuste entre as estruturas formais (legislação, planejamento e projetos governamentais) e as estruturas reais (divergências entre a administração pública e os segmentos de organizações particulares), no cenário brasileiro da política ambiental.

No Brasil, a Política Nacional do Meio Ambiente, criada em 1981, instituiu normas legais as quais, atualmente, todo empreendimento deve se submeter a fim de contribuir com o desenvolvimento sustentável do país (BRASIL, 1981), como a implantação de novas ferramentas de controle e fiscalização para os processos de desenvolvimento e atividades empresariais e suas implicações para o meio ambiente. Surgiu, portanto, na legislação brasileira os termos: Avaliação de Impacto Ambiental e Licenciamento Ambiental (SÁNCHEZ, 2008).

Uma das principais ferramentas para aplicação da legislação supracitada é o processo de Licenciamento Ambiental, orientado pela Resolução do Conselho Nacional do Meio Ambiente (CONAMA) no237/1997, que determinou a realização de estudos ambientais, para apresentar um levantamento local contemplando os meios socioeconômico, biótico e físico do ambiente, prevendo também os impactos ambientais e suas possíveis medidas mitigadoras ou compensatórias, as quais os empreendedores devem assumir o compromisso de cumprir em concomitância as ações de implantação e operação do empreendimento. Somente através deste procedimento, os futuros empreendimentos poderão ser instalados e operados com controle ambiental, mediante a concessão de licenças conferidas pelos órgãos do SISNAMA (BRASIL, 1997).

Os Termos de Referência dos órgãos ambientais orientam a realização de uma Avaliação de Impactos Ambientais (AIA) de forma a identificar, interpretar e valorar estes impactos a cerca de uma série de parâmetros de avaliação, a saber: "impactos diretos e indiretos; impactos benéficos e adversos; impactos temporários, permanentes e cíclicos; impactos imediatos, a médio e longo prazos; impactos reversíveis e irreversíveis; impactos locais, regionais e estratégicos" (BRASIL, 2012). E a Resolução CONAMA nº01/1986 ainda propôs que os impactos ambientais fossem avaliados com uma série de critérios, que avaliam o impacto com relação à sua magnitude e importância.

Prado Filho e Souza (2004) afirmaram que a AIA é considerada mundialmente como importante instrumento de gestão e proteção ambiental (BITAR e ORTEGA, 1998), principalmente quanto à indicação de ações preventivas que visam à sustentabilidade ambiental. Atualmente, porém, não existe nenhum instrumento legal regulamentado que determina uma metodologia padrão para que esta avaliação seja executada. Diante desta realidade, os estudos ambientais atuais são elaborados com uma grande variedade de métodos de avaliação de impacto ambiental, que podem ser mais generalistas ou detalhados, qualitativos ou quantitativos, o que permite, em determinados casos, que não haja clareza da dimensão de um determinado impacto oriundo de um empreendimento.

Diante deste cenário, o presente trabalho visa propor uma metodologia de ponderação de valores numéricos para atributos da Avaliação de Impacto Ambiental, a fim de mensurar a significância de cada impacto, tendo como referência principal a Resolução CONAMA nº 001/1986 (BRASIL, 1986), a fim de criar escalas para os critérios de magnitude e severidade do impacto através da combinação de atributos, além de verificar e testar a aplicação da metodologia proposta em estudo ambiental comparando com um método de AIA qualitativa. 


\section{MATERIAL E MÉTODOS}

Foram realizadas pesquisas bibliógraficas em artigos científicos e teses acadêmicas publicadas no período de 2000 a 2012 dentro da temática ambiental, bem, como a utilização de livros da bibliografia da temática do direito e da gestão ambiental, além de dados das legislações ambientais brasileiras. Outra ferramenta para pesquisa se deu com a busca do Sistema Integrado de Informações Ambientais (SIAM) e o site da Secretaria Estadual de Meio Ambiente e Desenvolvimento Sustentável (SEMAD).

Para base comparativa e aplicação de teste da metodologia ora proposta, foi utilizado o Estudo de Impacto Ambiental (EIA) do setor de mineração no estado de Minas Gerais, o qual utilizou uma metodologia de AIA qualitativa disposta em matrizes baseadas na metodologia de Leopold (1971).

\subsection{Levantamento de atributos para AIA}

Primeiramente, realizou-se o levantamento dos atributos de avaliação de impacto ambiental propostos pela Resolução CONAMA nº 01, de 23 de janeiro de 1986 (BRASIL, 1986). Em seguida, foi apontada a proposição da adoção de outros atributos de avaliação, com o intuito de tornar mais tangível à mensuração do critério de severidade de um impacto.

\subsubsection{Combinação de atributos}

Com a definição dos atributos adotados para avaliar os impactos, será realizada a combinação de alguns deles para a determinação do conceito de Severidade, sendo importante destacar que alguns conceitos avaliativos não são adequados para definir a importância de um impacto. Para isso, utilizar-se-á a metodologia de combinação de atributos (SÁNCHEZ, 2008), que determina os seguintes passos: definir os atributos que serão utilizados; estabelecer uma escala para cada um deles; e combiná-los mediante um conjunto de regras lógicas (critério de avaliação).

\subsubsection{Escala de classificação para Severidade e Magnitude}

Além da combinação de atributos, será elaborada uma escala para classificar o grau de severidade de um impacto, bem como para delimitar o alcance do impacto, definindo, portanto, o conceito de magnitude. A escala será composta por numeração de 1 a 5 , sendo que conforme a ascendência numérica, mais intensa classifica-se o grau dos critérios de severidade e magnitude. $\mathrm{O}$ modelo de escala proposta foi baseado nas escalas utilizadas em alguns Estudos de Impacto Ambiental, a saber: EIA Tejucana Mineração S.A (YKS, 2011), EIA TCLD GERDAU (YKS, 2010), EIA Mineração Fazenda Bota (SIGNUS VITAE, 2010).

\subsubsection{Procedimentos para ponderação de atributos}

Por fim, será elaborada uma equação matemática simplificada para aplicar a ponderação de valores numéricos conforme as bibliografias (TORQUETTI, 2001; RODRIGUES e CAMPANHOLA, 2003; BARBOSA, 2004; SANCHEZ, 2008). Para isso, conforme demonstrado na equação (1), cada atributo será assimilado a um valor numérico numa escala de 0 a 2 . Quanto mais elevado o valor, maior será seu grau de importância. Este valor será associado por meio de multiplicação de um peso (escala de 2 a 4) relativo a cada atributo que irá conferir o grau de importância do atributo.

$$
\sum^{\mathrm{Qa}}=(V a \times P)
$$


Sendo:

$\mathrm{Q} a=$ quantidade de atributos

$\mathrm{Va}=$ valor do atributo (Varia de 0 a 2. ).

$\mathrm{P}=$ valor do peso (Varia de 2 a 4 ).

Realizada todas as relações supracitadas, soma-se ao final o valor ponderado de cada atributo, que irá conferir a significância do impacto. Esta significância será determinada por uma escala de valores numéricos que também serão associados a cores para serem preenchidas na matriz de AIA. Sendo que os impactos serão classificados como: pouco significante significância média, significante, muito significante e significância crítica. Este somatório resultará em valores entre 11 e 30, e será criada um escala numérica, com atribuição de cores, para valorar por fim a Significância do impacto. Após a obtenção da avaliação final, os resultados poderão ser dispostos numa matriz que relacionam as ações do empreendimento com os aspectos e impactos ambientais.

\subsubsection{Elaboração de algoritmo}

Para sistematização e aplicação dos passos descritos acima, foi criado por meio do programa VisuAlg - Editor e Interpretador de Pseudocódigos um algoritmo que contempla todas as fases da metodologia ora apresentada, sendo que, após a inclusão dos dados solicitados, o programa fornece o resultado da significância do impacto avaliado e indica a cor relativa para o preenchimento da matriz de impactos. $\mathrm{O}$ algoritmo foi construído com a linguagem de programação apropriada ao programa supracitado e poderá ser disponibilizado em meio digital via contato com o autor.

\subsubsection{Preenchimento da Matriz de Avaliação de Impactos Ambientais.}

A matriz adotada para apresentação da Avaliação de Impacto Ambiental de um estudo ambiental (Erro! Fonte de referência não encontrada.) irá compilar o resultado da avaliação, juntamente às ações do empreendimento vinculadas aos aspectos que as mesmas desencadeiam, e por fim ao impacto gerado. Para isso, a mesma poderá ser construída no programa para construção de planilhas eletrônicas Microsoft Office Excel, e deverá ser preenchida conforme os procedimentos citados a seguir.

a. Listar cada ação proposta pelo empreendimento associado a sua fase de planejamento, implantação, operação e encerramento.

b. Listar todos os aspectos ambientais que estão relacionados às ações propostas pelo empreendimento;

c. Marcar com " $X$ " cada quadrícula correspondente a uma ação relacionando-a a um aspecto ambiental;

d. Apresentar todos os impactos ambientais oriundos pelo empreendimento, dividindo entre os meios associados: físico, biótico e socioeconômico;

e. Preencher a quadrícula que relaciona o aspecto ambiental com o impacto que causará com a referida cor da significância pré - avaliada do determinado impacto. 


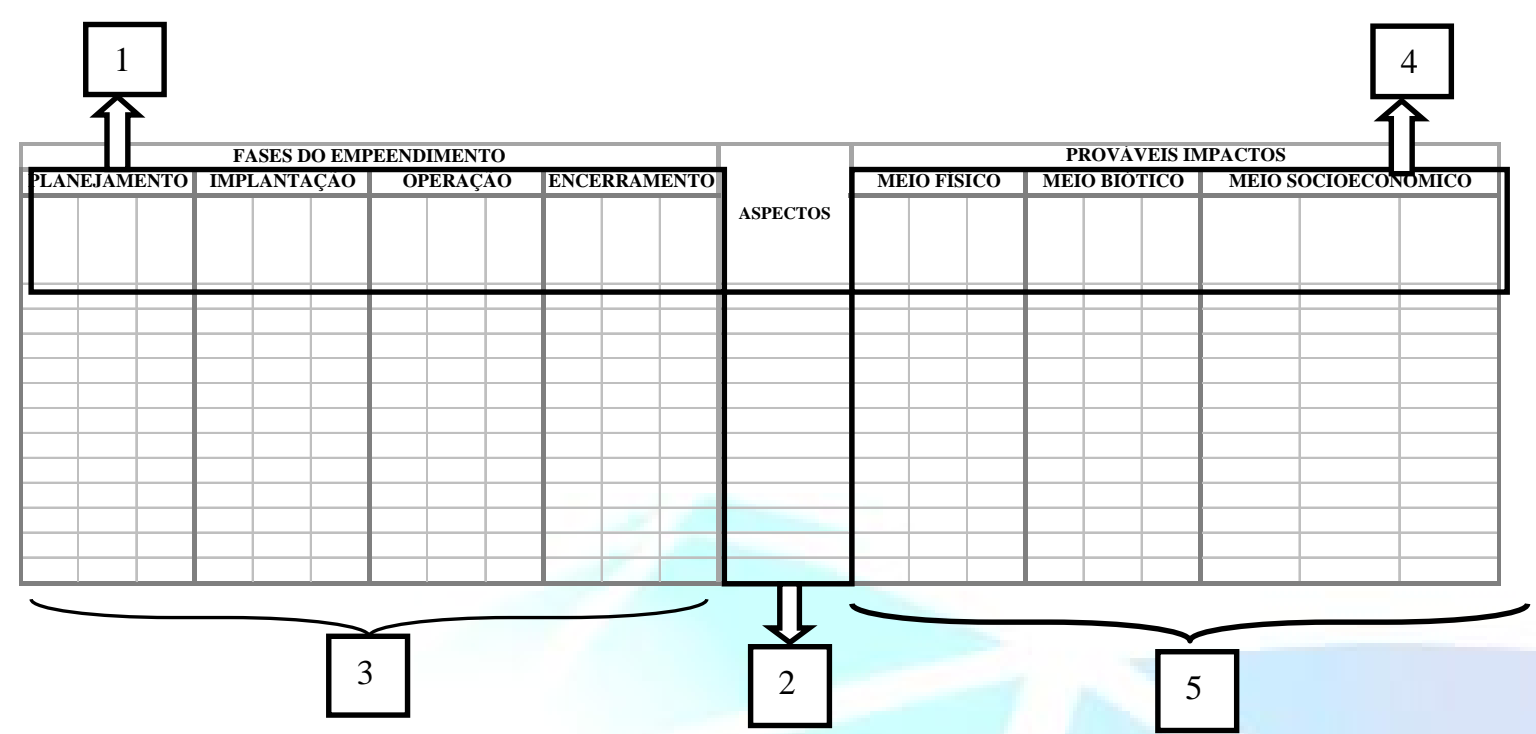

Figura 1. Modelo de Matriz de Avaliação de Impactos Ambientais. Fonte: Adaptado YKS, 2010 e YKS, 2011.

\subsubsection{Teste do modelo}

Para testar a metodologia quantitativa para valoração da significância de um impacto, buscou-se uma AIA aplicada no EIA do empreendimento Tejucana Mineração S/A, cuja metodologia utilizada neste caso era apenas qualitativa. A partir da matriz preenchida com as mesmas ações e impactos, será refeita a AIA com o método quantitativo proposto e posteriormente serão comparados os resultados para verificar a eficácia do modelo.

\section{RESULTADOS E DISCUSSÃO}

Inicialmente, adotaram-se as proposições da Resolução CONAMA n 01 , de 23 de janeiro de 1986 (BRASIL, 1986), preconizando que a análise do impacto ambiental seja realizada através da identificação da magnitude e da importância do impacto, sendo que para classificar a importância são avaliados os seguintes atributos do impacto: imediato, médio ou longo prazo; temporário ou permanente; reversível ou irreversível; cumulativo sinergético ou não cumulativo.

Além dos atributos supracitados, serão adicionados os atributos que avaliam: se o impacto ambiental atinge um indicador protegido ou não por legislação; se o impacto causa modificação em determinado indicador ambiental; e a probabilidade de ocorrência do impacto. Estes últimos atributos visam aprimorar a definição da severidade do impacto.

Ressalta-se que os critérios de expressão e origem, a saber: positivo / negativo e direto / indireto, apesar de indicados pela Resolução citada, não serão utilizados para a classificação da severidade, visto que, conforme exposto por Sanchez (2008), estes atributos não tornam os impactos menos ou mais significativos. Ou seja, são atributos utilizados na AIA, mas neste caso, não aplicados à mensuração da significância.

Para definição do conceito de severidade, foram combinados atributos referentes aos critérios de: Reversibilidade, Duração, Indicador com proteção legal e Modificação (Erro! Fonte de referência não encontrada.), que em conjunto possibilitam a classificação de cinco estágios distintos de severidade.

Com o exposto, apresentam-se as combinações de critérios para classificação da severidade de um impacto (Tabela ). Torna-se importante salientar que o impacto classificado como 
irreversível, inerentemente também será considerado permanente. O que agrega um grau de severidade mais alto, não sendo necessário avaliar se o impacto altera o indicador ambiental protegido, ou não, por lei.

Para definição do critério de magnitude foram propostas cinco classes que caracterizam o alcance do impacto ambiental, conforme apresentado na Tabela 2.

Quadro 1 - Critérios adotados para a caracterização da Severidade de um impacto

$\begin{array}{llll}\text { Critério } & \text { Atributo } & \text { Sigla } & \text { Definição }\end{array}$

Reversível

Reversibilidade

Irreversível

Temporário

Duração

Permanente
O indicador ambiental afetado não retorna às $\mathrm{R}$ condições originais uma vez cessadas a ação geradora do impacto. *.

O indicador ambiental não retorna à condição original quando cessada a ação. *.

$\mathrm{T}$

O efeito do impacto se manifesta somente durante a atividade geradora. *.

O efeito do impacto se manifestar numa $\mathrm{Pe} \quad$ escala em longo prazo (anos), ainda que interrompida a atividade geradora. ${ }^{*}$

\begin{tabular}{|c|c|c|c|}
\hline \multirow{3}{*}{$\begin{array}{c}\text { Alteração em } \\
\text { indicador } \\
\text { ambiental } \\
\text { protegido }\end{array}$} & $\begin{array}{l}\text { Não altera indicador } \\
\text { ambiental }\end{array}$ & $\mathrm{X}$ & $\begin{array}{l}\text { O impacto não altera o indicador ambiental } \\
\text { com, ou sem proteção legal. }\end{array}$ \\
\hline & Altera indicador ambiental & Y & $\begin{array}{l}\text { O impacto altera o indicador ambiental sem } \\
\text { proteção legal. }\end{array}$ \\
\hline & $\begin{array}{l}\text { Altera indicador ambiental } \\
\text { protegido }\end{array}$ & Z & $\begin{array}{l}\text { O impacto altera o impacto ambiental com } \\
\text { proteção legal }\end{array}$ \\
\hline \multirow{2}{*}{$\begin{array}{l}\text { Modificação } \\
\text { da realidade } \\
\text { local }\end{array}$} & Modificador & MM & $\begin{array}{l}\text { O impacto modifica a realidade local dos } \\
\text { aspectos socioeconômicos, dos aspetos } \\
\text { físicos e da flora e fauna. }\end{array}$ \\
\hline & Não Modificador & NM & $\begin{array}{l}\text { O impacto não modifica a realidade local dos } \\
\text { aspectos socioeconômicos, dos aspetos } \\
\text { físicos e da flora e fauna. }\end{array}$ \\
\hline
\end{tabular}

* Definições adaptadas de Sanchéz, 2008, p.292. 
Tabela 1 - Combinação de atributos para a caracterização do critério Severidade de um impacto

$\begin{array}{llll}\text { Atributo Sigla } & \text { Definição } & \text { Combinação } & \text { Valor }\end{array}$

Severidade

baixa

S1

O impacto é reversível, temporário, não altera indicador ambiental e não modifica a

realidade local dos aspectos socioeconômicos, dos aspetos físicos e da flora e fauna.

O impacto é reversível, temporário, altera

Severidade

pequena

S2 indicador ambiental sem proteção legal e não modifica a realidade local dos aspectos socioeconômicos, dos aspetos físicos e da

R T Y NM

0,8 flora e fauna.

R T X NM

0,4
O impacto é reversível, temporário, altera

Severidade S3 indicador ambiental com proteção legal e não média modifica a realidade local dos aspectos socioeconômicos, dos aspetos físicos e da flora e fauna.

O impacto é reversível, temporário, altera indicador ambiental com proteção legal e modifica a realidade local dos aspectos socioeconômicos, dos aspetos físicos e da flora e fauna.

Severidade S4 ou

O impacto é irreversível, permanente, altera indicador ambiental com ou sem proteção legal e não modifica a realidade local dos aspectos socioeconômicos, dos aspetos físicos e da flora e fauna.

O impacto é irreversível, permanente, altera indicador ambiental com ou sem proteção

Severidade crítica legal e modifica a realidade local dos aspectos
R T Z NM 1,2

R T Z MM

ou

1,6

Ir Pe (X/Y/Z) NM

Ir Pe (X/Y/Z) MM

2 socioeconômicos, dos aspetos físicos e da

flora e fauna. 
Tabela 2 - Critérios adotados para a caracterização da magnitude de um impacto.

\begin{tabular}{|c|c|c|c|}
\hline Critério & Sigla & Definição & Valor \\
\hline Magnitude localizada & M1 & $\begin{array}{l}\text { O alcance do impacto se limita ao local } \\
\text { de origem do impacto, ou seja, atinge a } \\
\text { área diretamente afetada (ADA) }\end{array}$ & 0,4 \\
\hline Magnitude pequena & M2 & $\begin{array}{l}\text { O alcance do impacto se limita a área de } \\
\text { inserção do empreendimento. Atinge a } \\
\text { equipe de funcionários atuantes no } \\
\text { empreendimento. }\end{array}$ & 0,8 \\
\hline Magnitude média & M3 & $\begin{array}{l}\text { O alcance do impacto extrapola os } \\
\text { limites do empreendimento e atinge área } \\
\text { de influência direta (AID). }\end{array}$ & 1,2 \\
\hline Magnitude grande & M4 & $\begin{array}{l}\text { O alcance do impacto extrapola os } \\
\text { limites do empreendimento e atinge a } \\
\text { área de influência indireta (AII). }\end{array}$ & 1,6 \\
\hline $\begin{array}{l}\text { Magnitude muito } \\
\text { grande }\end{array}$ & M5 & $\begin{array}{l}\text { O alcance do impacto extrapola os } \\
\text { limites do empreendimento e extrapola a } \\
\text { área de influência indireta (AII) }\end{array}$ & 2 \\
\hline
\end{tabular}

Com todos os atributos de avaliação de impacto definidos, será proposto à atribuição de valores para cada um destes critérios a fim de definir um grau de significância ao impacto. Para mensurar a significância dos impactos ambientais, foi proposto para cada atributo de avaliação de impacto a assimilação dos valores de 0 a 2 , sendo que estes serões atribuídos a três pesos distintos, sendo que os atributos relativos à probabilidade de ocorrência possuem peso igual a 4; os atributos relativos à escala temporal têm peso igual a 2 e os atributos relativos à cumulatividade, à severidade e à magnitude têm peso igual a 3 (Tabela 3 ).

Ao final, a soma dos valores ponderados representa a significância do impacto que será expressa conforme valores e representação através de cores baseado em Signos Vitae (2010), como apresentado na Tabela 4. 
Tabela 3 - Ponderação de valores

\begin{tabular}{|c|c|c|c|c|}
\hline Critério & Atributo & $\begin{array}{l}\text { Valor do } \\
\text { atributo }\end{array}$ & Peso & $\begin{array}{c}\text { Valor ponderado } \\
(\text { Va } \times 1)\end{array}$ \\
\hline \multirow{2}{*}{$\begin{array}{l}\text { Probabilidade } \\
\text { de ocorrência }\end{array}$} & Ocorrência certa & 2 & \multirow[b]{2}{*}{4} & 8 \\
\hline & Ocorrência incerta / baixa probabilidade & 1 & & 4 \\
\hline \multirow{2}{*}{$\begin{array}{l}\text { Escala } \\
\text { temporal }\end{array}$} & Imediata & 1 & \multirow{2}{*}{2} & 2 \\
\hline & Médio prazo / Longo prazo & 2 & & 4 \\
\hline \multirow[b]{2}{*}{ Cumulatividade } & Cumulativo & 2 & \multirow{2}{*}{3} & 6 \\
\hline & Não cumulativo & 1 & & 3 \\
\hline \multirow{5}{*}{ Severidade } & Severidade baixa & 0,4 & \multirow{5}{*}{3} & 1,2 \\
\hline & Severidade pequena & 0,8 & & 2,4 \\
\hline & Severidade média & 1,2 & & 3,6 \\
\hline & Severidade grande & 1,6 & & 4,8 \\
\hline & Severidade crítica & 2 & & 6 \\
\hline \multirow{5}{*}{ Magnitude } & Localizada & 0,4 & \multirow{5}{*}{3} & 1,2 \\
\hline & Pequena & 0,8 & & 2,4 \\
\hline & Média & 1,2 & & 3,6 \\
\hline & Grande & 1,6 & & 4,8 \\
\hline & Muito grande & 2 & & 6 \\
\hline
\end{tabular}

Tabela 4 - Valores de significância

\begin{tabular}{ccc}
\hline Valor & Cor & Significância \\
\hline 11 a 15 & Verde & Impacto pouco significativo \\
\hline 16 a 20 & Bege & Impacto média significância \\
21 a 24 & Amarelo & Impacto significativo \\
25 a 27 & Laranja & Impacto muito significativo \\
28 a 30 & Vermelho & Impacto chave /crítico \\
\hline
\end{tabular}




\subsection{Teste do modelo}

As Figura 2 e 3 apresentam, respectivamente, a matriz de AIA do EIA de um empreendiemnto, e a matriz resultante com a aplicação do modelo de avaliação da significância proposto. Foi realizada a avaliação da significância de 3 impactos ambientais para cada meio (físico, biótico e socioeconômico), considerando a fase de instalação e operação do EIA do empreendimento Tejucana Mineração S/A. Desta forma, obtiveram-se 33 avaliaçãoes sendo apresentados os resultados expressos na Tabela 5.

Os resultados demonstram que a aplicação da metodologia quantitativa tornou de forma geral a significância dos impactos avaliados mais elevada. Em todos os meios avaliados, pode-se perceber o crescimento do nível de significância dos impactos. Para o meio físico e biótico verificou-se o crescimento em $45 \%$ dos impactos avaliados em um nível de significância, já para o meio socioeconômico, constatou-se que houve aumento em $82 \%$ dos impactos avaliados em dois níveis da significância, o que se pode dizer que este método torna a avaliação da significância mais severa para o meio socioeconômico (Tabela 5).

Dentre as 33 avaliações realizadas, apenas um obteve um resultado com menor grau de significância, representando apenas $3 \%$ do todo, o que mais uma vez demonstra que o método quantitativo ora apresentado pode ser classificado como mais rígido.

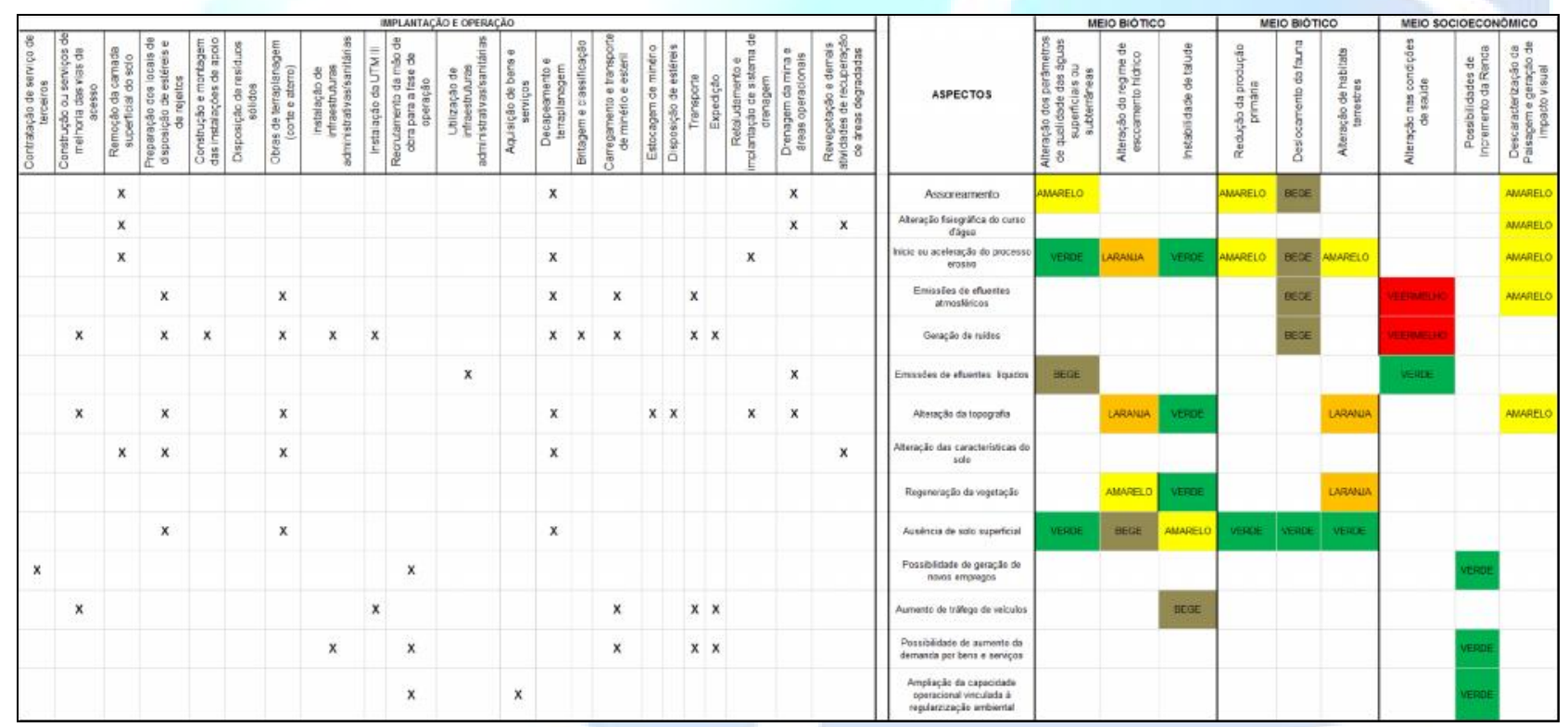

Figura 2. Matriz de AIA Tejucana Mineração S.A. Fonte: Adaptado de EIA Tejucana Mineração S.A, 2011. 


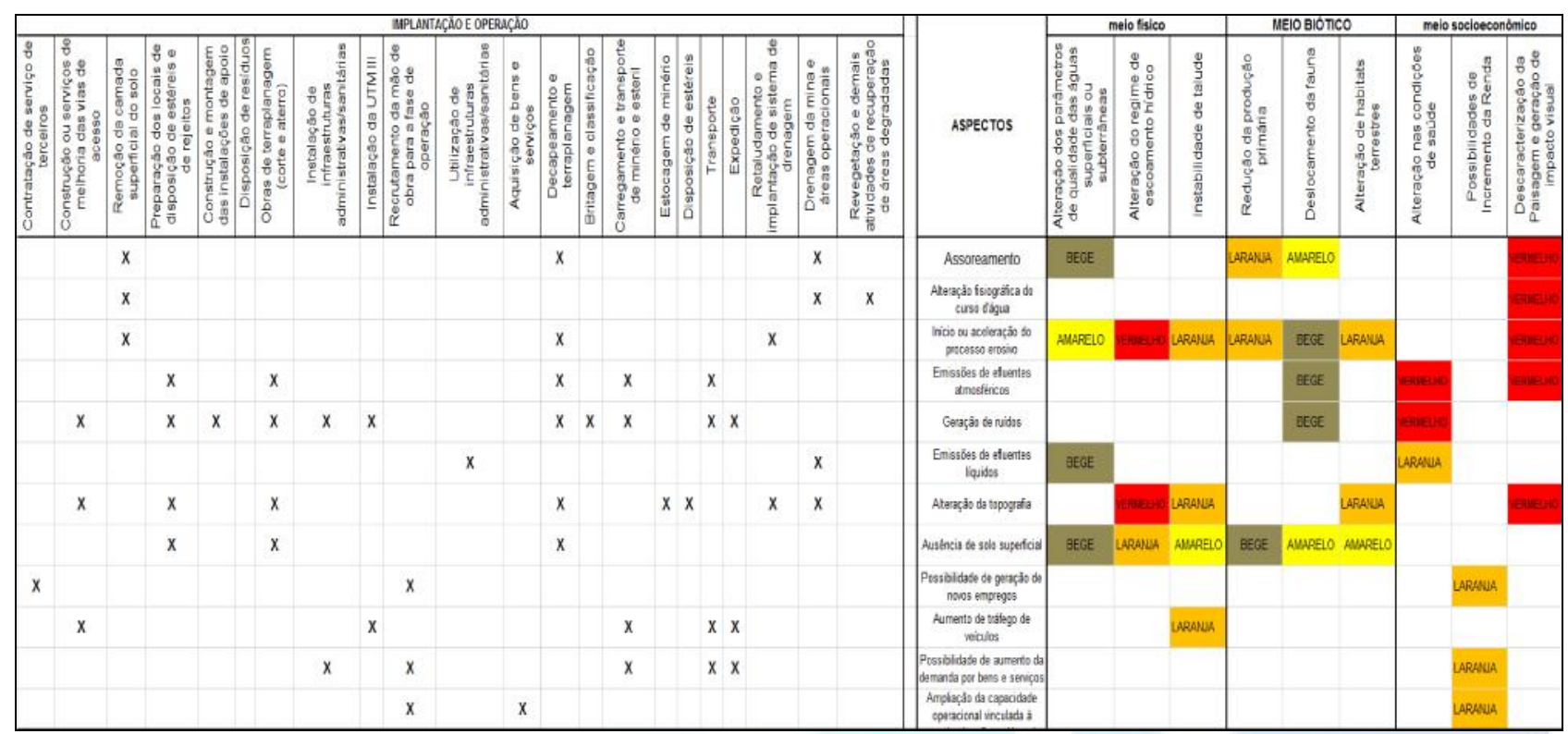

Figura 3. Matriz de Significância de AIA - Teste do modelo

Tabela 5 - Análise comparativa do método qualitativo para avaliação da Significância.

\begin{tabular}{|c|c|c|c|c|c|c|}
\hline & \multirow{3}{*}{$\begin{array}{c}\mathrm{N}^{\mathbf{o}} \text { de } \\
\text { impactos } \\
\text { avaliados }\end{array}$} & \multirow{3}{*}{$\begin{array}{l}\text { Resultados } \\
\text { iguais }\end{array}$} & \multicolumn{4}{|c|}{ Resultados diferentes } \\
\hline & & & \multicolumn{2}{|c|}{ Aumento da Significância } & \multicolumn{2}{|c|}{$\begin{array}{l}\text { Diminuição da } \\
\text { Significância }\end{array}$} \\
\hline & & & $\begin{array}{l}\text { Um nível } \\
\text { acima }\end{array}$ & $\begin{array}{l}\text { dois níveis } \\
\text { acima }\end{array}$ & $\begin{array}{l}1 \text { nível } \\
\text { abaixo }\end{array}$ & $\begin{array}{l}2 \text { níveis } \\
\text { abaixo }\end{array}$ \\
\hline \multicolumn{7}{|c|}{ Análise para o Meio Físico } \\
\hline Valor absoluto & 11 & 2 & 5 & 3 & 1 & 0 \\
\hline Porcentagem & $100 \%$ & $18 \%$ & $45 \%$ & $27 \%$ & $9 \%$ & $0 \%$ \\
\hline \multicolumn{7}{|c|}{ Análise para o Meio Biótico } \\
\hline Valor absoluto & 11 & 3 & 5 & 2 & 0 & 0 \\
\hline Porcentagem & $100 \%$ & $27 \%$ & $45 \%$ & $18 \%$ & $0 \%$ & $0 \%$ \\
\hline \multicolumn{7}{|c|}{ Análise para o Meio Socioeconômico } \\
\hline Valor absoluto & 11 & 2 & 0 & 9 & 0 & 0 \\
\hline Porcentagem & $100 \%$ & $18 \%$ & $0 \%$ & $82 \%$ & $0 \%$ & $0 \%$ \\
\hline \multicolumn{7}{|c|}{ Análise geral } \\
\hline Valor absoluto & 33 & 7 & 10 & 14 & 1 & 0 \\
\hline Porcentagem & $100 \%$ & $21 \%$ & $30 \%$ & $42 \%$ & $3 \%$ & $0 \%$ \\
\hline
\end{tabular}

Alguns autores apontam que a valoração e quantificação de impactos ambientais podem ser reconhecidas como uma fragilidade da AIA vista a dificuldade de se atribuir valor para certos elementos, sendo que ainda não existem métodos, normas ou padrões para tal (OLIVEIRA e BURSZTYNB, 2001), ao passo que se pode também verificar que a não quantificação da significância dos impactos pode subestimar sua importância, o que poderia futuramente acarretar em medidas de mitigação, compensação ou potencializarão não satisfatórias ou ineficazes para ao contexto real. 


\section{Comparação da Significância para o Meio Físico}

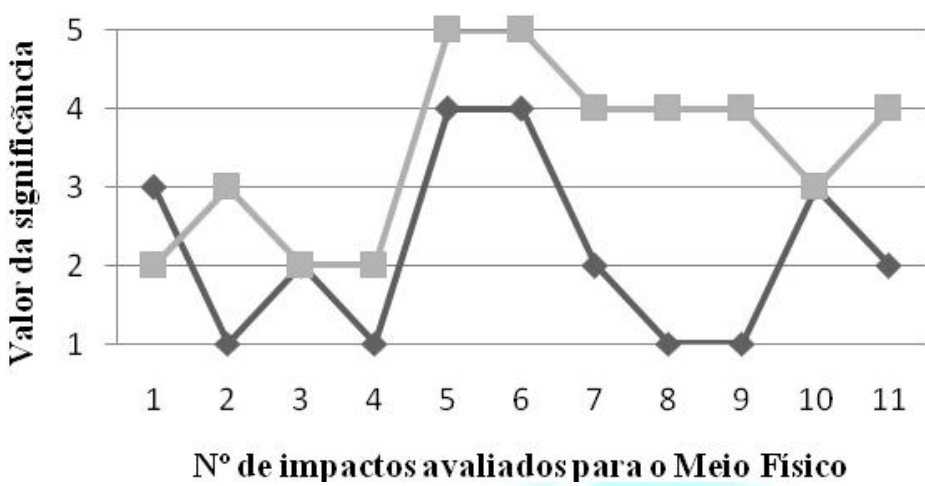

— Siginificância método qualitativo

- Significância método qualitativo

$\mathrm{N}^{\circ}$ de impactos avaliados para o Meio Físico

Figura 4. Comparação dos métodos qualitativo e quantitativo para avaliação da Significância para o Meio Físico

\section{Comparação da Significância para o Meio Biótico}

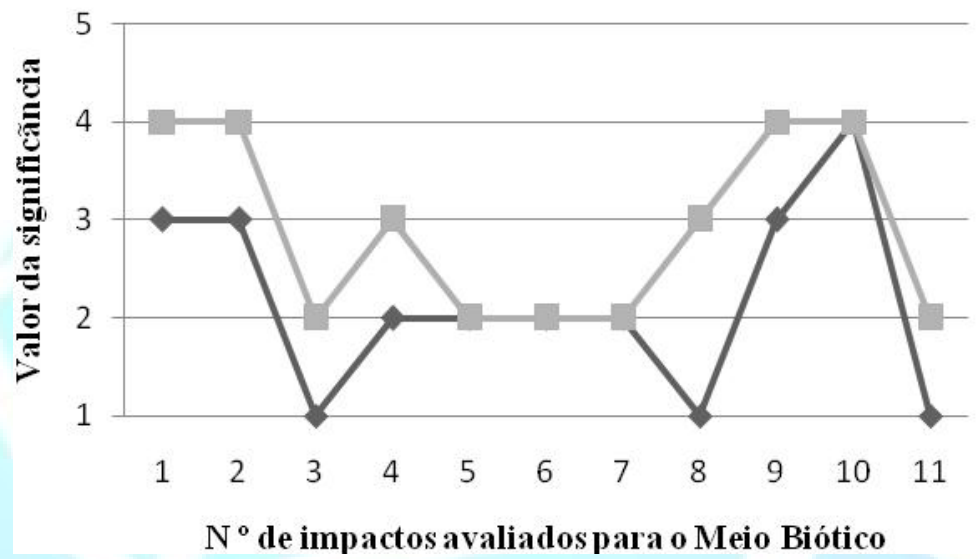

— Significância método qualitativo

- Significância método qualitativo

Figura 5. Comparação dos métodos qualitativo e quantitativo para avaliação da Significância para o Meio Biótico

\section{Comparação da Significância para o Meio Socioeconômioco}

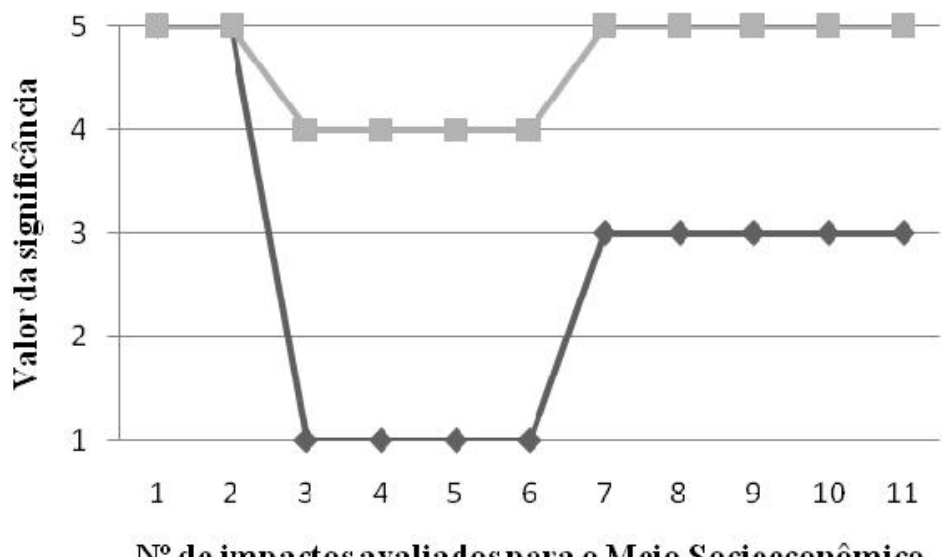

$\multimap$ Significância método qualitativo

- Significância método qualitativo

Figura 6. Comparação dos métodos qualitativo e quantitativo para avaliação da Significância para o Meio Socioeconômico 
As Figuras 4 a 6 demonstram o aumento do grau da significância dos impactos avaliados com a aplicação do modelo quantitativo. Avalia-se, portanto que, sendo um modelo de resultados mais severos poderá não ser adotado por determinados empreendimentos. Em contrapartida, esta rigidez conferida a esta metodologia pode ser transformada em benefícios aos aspectos ambientais impactados, visto a necessidade das ações de controle ambiental que deverão ser tomadas baseadas nestes resultados.

Carvalho e Lima (2010) ressaltaram que as diferentes formas existentes para a avaliação de impacto ambiental têm gerado grandes debates científicos na busca por métodos cada vez melhores para a definição correta dos impactos, visando a preservação ambiental e o desenvolvimento sustentável.

Deve-se considerar, porém, as limitações do conhecimento científico no estabelecimento de impactos potenciais. Ou seja, diante do exposto não se pode garantir que apenas a utilização deste método seja satisfatória para sanar todas as falhas existentes acerca da AIA sendo importante considerar que a aplicação do método seja realizada por equipe multidisciplinar. Também deve ocorrer inovações para aplicação da AIA, a fim de que cada impacto vinculado a um dado meio, seja avaliado com suas características intrínsecas , aproximando-se ao máximo da situação real. Isto é de extrema importância para a validade do dado analisado que será valorado através de um peso.

Rodrigues e Campanhola (2003) enfatizaram que um dos requisitos mais importantes para a eficácia do método quantitativo de AIA proposto em seus estudos é a coleta de informações confiáveis quanto à situação do empreendimento. Torquetti (2001) expõe que um ponto crítico do planejamento do Sistema de Gestão Ambiental (SGA) nos moldes da Norma Brasileira (NBR) ISO 14.001(ABNT, 1996) recai sobre a tarefa de avaliação da importância ou significância dos impactos ambientais. Como esta tarefa fornece a base para o estabelecimento e revisão dos objetivos e metas ambientais em cada empresa, atenção especial deve ser dada à mesma, para que o SGA realmente seja implementado com sucesso.

Assim, pode-se considerar que o método quantitativo de avaliação da significância poderá, além de ser aplicada para estudos de impacto ambiental (EIA), também poderá ser aplicada como método de identificar indicadores ambientais para prevenção aos danos ambientais. A possibilidade de apresentar os resultados da AIA em gráficos, torna-se também um instrumento de análise e síntese para o planejamento e a gestão ambiental de um empreendimento.

\section{CONCLUSÕES}

A metodologia de ponderação de valores e agregação de atributos para a avaliação da significância dos impactos ambientais de um empreendimento se mostra pertinente ao que tange os objetivos propostos pela legislação ambiental vigente que apresenta os critérios de avaliação de impactos para os estudos ambientais. Ao utilizar os atributos propostos e a agregação de valores numéricos almeja-se diminuir o nível de subjetividade da AIA, sendo que com os resultados expostos, afirma-se que esta metodologia se adéqua à elaboração de estudos ambientais que se submetem ao processo de licenciamento ambiental.

Conclui-se, contudo, que os objetivos propostos neste trabalho foram alcançados e demonstraram-se aplicáveis ao se analisar um estudo de caso comparativo entre uma metodologia quantitativa e outra qualitativa. É importante considerar que mesmo a aplicação de um método que objetiva enquadrar a AIA através de pesos, ainda assim deve-se considerar a existência da subjetividade, visto que a análise poderá apresentar resultados distintos de um avaliador para outro. Logo, a junção de métodos qualitativos ou quantitativos e outras técnicas, conferem maior aproximação com a realidade e confiabilidade à AIA. 
Por fim, pode-se também verificar que muito ainda tem-se que aprimorar acerca da temática da Avaliação de Impactos Ambientais para torná-la cada vez mais uma ferramenta eficaz tanto para estudos ambientais, como para o Sistema de Gestão Ambiental.

\section{REFERÊNCIAS}

AGNES, C. C.; CALEGARI, L.; GATTO, D. A.; STANGERLIN, D. M. Uma discussão sobre a descentralização da gestão ambiental. Revista Científica Eletrônica De Engenharia Florestal. São Paulo, n. 14. 53-73 p. ago. 2009. Disponível em: <http://www.admpg.com.br/revista2012a/artigos/artigos/Ambiental/14-01339356891. pdf>. Acesso: em: 2 nov.2012.

ASSOCIAÇÃO BRASILEIRA DE NORMAS TÉCNICAS - ABNT. NBR ISO 14001. Sistemas de gestão ambiental - Especificação e diretrizes para uso. Rio de Janeiro. 14 p. out. 1996.

BARBOSA, T.A.S. Análise do estudo de impacto ambiental da PCH ninho da água proposta de otimização do processo de licenciamento ambiental utilizando uma matriz simplificada. Dissertação de Mestrado, Pós-Graduação em Engenharia da Energia, - Universidade Federal de Itajubá. Itajuba, $2004.119 \mathrm{p}$.

BRASIL. Conselho Nacional de Meio Ambiente. Resolução nº 001, de 23 de março de 1986. Estabelece as definições, as responsabilidades, os critérios básicos e as diretrizes gerais para uso e implementação da Avaliação de Impacto Ambiental como um dos instrumentos da Política Nacional do Meio Ambiente. Diário Oficial da União, Brasília, DF. 2548-254917p. fev.1986.

BRASIL. Conselho Nacional de Meio Ambiente. Resolução nº 273, de 19 de dezembro de 1997. Dispõe sobre licenciamento ambiental; competência da União, Estados e Municípios; listagem de atividades sujeitas ao licenciamento; Estudos Ambientais, Estudo de Impacto Ambiental e Relatório de Impacto Ambiental. Diário Oficial da União, Brasília, DF. 20 -23 p. 22. dez. 1997.

BRASIL. Lei $n^{\circ}$ 6.938, de 31 de agosto de 1981. Dispõe sobre a Política Nacional do Meio Ambiente, seus fins e mecanismos de formulação e aplicação, e dá outras providências. Diário Oficial da União, Brasília, DF. 18509 p. 02. set. 1981.

BRASIL. Secretaria de Estado de Meio Ambiente e Desenvolvimento Sustentável - SEMAD.

Termo de Referência geral para elaboração de Estudo de Impacto Ambiental (EIA) /Relatórios de Impacto Ambiental - RIMA. p.51. Disponível em:

<http://www.meioambiente.mg.gov.br/noticias/1/1167-termos-de-referencia-para-elaboracao-deestudo-de-impactorelatorio-de-impacto-ambiental-eiarima> Acesso em: 3 nov. 2012.

CARVALHO, L.D.; LIMA, A.V. Metodologias para avaliação de impactos ambientais de aproveitamentos hidrelétricos. ENCONTRO NACIONAL DE GEÓGRAFOS. - CRISE, PRÁXIS E AUTONOMIIA: ESPAÇOS DE RESISTÊNCIA E DE ESPERANÇAS. ASSOCIAÇÃO DE GEÓGRAFOS BRASILEIRO. ENG., 16., Anais... Porto Alegre. p. 11, 2010.

DECLARAÇÃO do Rio de Janeiro sobre Meio Ambiente e Desenvolvimento. Conferência das Nações Unidas sobre Meio Ambiente e Desenvolvimento. Rio de janeiro. p.153 - 159. 1992. 
LEOPOLD, L. B. A procedure for evaluating environmental impact. v.28 . n. 2. 13 p. US Dept. of the Interior, 1971.

MILARÉ, E.; COIMBRA, J. A. A. Antropocentrismo x Ecocentrismo na ciência jurídica. Revista de Direito Ambiental, São Paulo: ed. RT (Revista dos Tribunais), n. 36, p.9 - 42, out.- dez. 2004.

MOREIRA, I.V.D. Vocabulário Básico de Meio Ambiente. Rio de Janeiro, Fundação Estadual de Engenharia do Meio Ambiente do Rio de Janeiro (FEEMA/RJ) e Petrobrás. 4 ed. 13 p.1992.

MORGAN, R.K. Environmental Impact Assessment. Dordbrecht: Kluwer Academic Publishers, 1998, $307 \mathrm{p}$.

MORRIS, P.; THERIVEL, R. Methods of Environmental Impact Assessment: the natural and built environmental series 2. Londres: UCL Press Limited, 1995. 378p.

OLIVEIRA, A.A.; BURSZTYNB, M. Avaliação de impacto ambiental de políticas públicas. Revista Internacional de Desenvolvimento Local, v.2, n.3. p. 45-56. set. 2001.

ROCHA, C.A.; CANTO, J.L.; PEREIRA, P.C. Avaliação de Impactos Ambientais nos Países do Mercosul. Ambiente \& Sociedade, v.8, n.2. p. 148-160.2005.

RODRIGUEZ, G.S.; CAMPANHOLA, C. Sistema integrado de avaliação de impacto ambiental aplicado a atividades do Novo Rural. Pesquisa Agropecuária Brasileira , Brasília, v.38, n.4, p.445-451, 2003.

PRADO FILHO, J.F.; SOUZA, M.P. O licenciamento ambiental da mineração no Quadrilátero Ferrífero de Minas Gerais: uma análise da implementação de medidas de controle ambiental formuladas em EIAs/RIMAs. Engenharia Sanitária e Ambiental.[online]. v.9, n.4, p.343-349, 2004. Disponível em: <http://www.scielo.br/scielo.php?pid=S1413$41522004000400012 \&$ script=sci_abstract\&tlng=pt>. Acesso em: 30 0ut. 2012.

SANCHES, L. E. Avaliação de impacto ambiental: conceitos e metas. São Paulo. Oficina de textos, 2008. 495 p.

SIGNUS VITAE. Manual de identificação e avaliação dos impactos do empreendimento: Estudo de Impacto ambiental EIA/RIMA Mineração Fazenda Bota ICAL Indústria de Calcinação Ltda. p.10.2010.

STAMM, H.R. Método para Avaliação de Impacto Ambiental (AIA) em projetos de grande porte: estudo de caso de uma usina termelétrica. $281 \mathrm{f}$. 2003. Tese de doutorado (Tese), Programa de Pós-Graduação em Engenharia Industrial. Universidade Federal de Santa Catarina. Florianópolis, SC. 2003.

SOUSA, W.L. Impacto ambiental de hidrelétricas: uma análise comparativa de duas abordagens. 154f. 2000. Tese de doutorado (Doutorado) - Universidade Federal do Rio de Janeiro, 2000 . 
TORQUETTI, Z.S.C. Planejamento do sistema de gestão ambiental - SGA - nas pequenas e médias industriais. $\mathbf{2 1}^{\circ}$ Congresso Brasileiro de Engenharia Sanitária e Ambiental. Anais.. Disponível em: <http://www.bvsde.paho.org/bvsaidis/brasil21/vi-060.pdf>. Acesso em: 2 nov. 2012

WATHERN, P. An introductory guide to EIA. In: Wathern, P. (Ed.). Environmental Impact Assessment. Theory and Practic. London, Unwin Hyman. 35 p.1998.

WESTSMAN, W.E. Ecology, Impact Assesssment, and Enviorenmental Planning. New York: Wiley. 544 p. 1985 .

YKS Serviços. Estudo de Impacto Ambiental do Transportadores de Correia de Longa Distância TCLD da GERDAU. Miguel Burnier, MG. p.314. 2010.

YKS Serviços. Estudo de Impacto Ambiental da Tejucana Mineração S.A. Brumadinho, MG. p. 534. 2011 